\title{
Effect of aerobic exercise on lipaemia and its fatty acid profile after a meal of moderate fat content in eumenorrhoeic women
}

\author{
Nikos Kokalas, Anatoli Petridou, Michalis G. Nikolaidis and Vassilis Mougios* \\ Department of Physical Education and Sport Science, Aristotle University of Thessaloniki, 54124 Thessaloniki, Greece \\ (Received 16 February 2005 - Revised 10 May 2005 - Accepted 24 May 2005)
}

\begin{abstract}
Exercise prior to eating has repeatedly been shown to reduce postprandial lipaemia. The aim of the present study was to investigate whether this effect was manifest in the presence of two factors that independently mitigate postprandial lipaemia: eumenorrhoea and moderate fat intake. Eight healthy eumenorrhoeic rowers consumed a meal of moderate fat content (35\% total energy, $0.66 \mathrm{~g} / \mathrm{kg}$ body mass) $14 \mathrm{~h}$ after having either rowed at $55 \%$ of maximal aerobic power $(81 \%$ of maximal heart rate) for $80 \mathrm{~min}$ or rested. Both trials were performed during the luteal phase. Blood samples were drawn before the meal and for $8 \mathrm{~h}$ postprandially for the measurement of individual fatty acids in the triacylglycerol and NEFA fractions, as well as of glucose, insulin and oestradiol. Plasma oestradiol concentrations were not significantly different in the two trials. The postprandial lipaemic response, expressed as either plasma triacylglycerol concentration or area under the triacylglycerol-time curve, was $35 \%$ lower $(P<0 \cdot 05)$ after exercise. The postprandial glycaemic and insulinaemic responses were also lower, indicating increased insulin sensitivity, whereas the NEFA response was higher, suggesting a lower entrapment of dietary fatty acids in adipose tissue after exercise. Finally, exercise increased the proportion of unsaturated:saturated NEFA during the postprandial period. In conclusion, aerobic exercise performed $14 \mathrm{~h}$ before a moderate-fat meal reduced postprandial lipaemia in women in the luteal phase. This effect shows the potential of exercise to mitigate even moderate lipaemic responses in eumenorrhoeic women.
\end{abstract}

Oestradiol: Insulin: Postprandial lipaemia: Triacylglycerols

There is substantial evidence that a high postprandial concentration of plasma triacylglycerols (TG) is an independent risk factor for coronary artery disease (Karpe, 1999; Gill \& Hardman, 2003). Given that individuals in developed countries usually spend most of their time in an absorptive state, considerable research interest has been directed towards elucidating the factors that influence the postprandial lipaemic response. Fasting plasma TG concentration, meal composition, meal time, diet, obesity, body fat distribution, oestrogen concentration and physical exercise have all been identified as having an influence (Romon et al. 1997; Koutsari et al. 2001; Pirro et al. 2001; Kolifa et al. 2004). With regard to the latter two factors in particular, endogenous and exogenous oestrogens have been associated with reduced postprandial lipaemia (Westerveld, 1998; Van Beek et al. 1999), whereas physical exercise has repeatedly been shown to decrease postprandial lipaemia, especially when performed on the day before a high-fat meal (e.g. Aldred et al. 1994; Tsetsonis \& Hardman, 1996a,b; Tsetsonis et al. 1997; Petitt et al. 2003).

The vast majority of studies from other laboratories have investigated the effect of exercise on postprandial lipaemia by using meals of high fat content (at least $1 \mathrm{~g} / \mathrm{kg}$ body mass and over $60 \%$ total energy). This greatly exceeds usual - let alone recommended - fat intakes and may exaggerate the observed differences between exercise and rest. We have recently conducted a study in healthy males, in which we examined the effect of prior exercise on lipaemia after a meal with a composition closer to that of a typical Western diet (Kolifa et al. 2004). We found that exercise did decrease postprandial lipaemia, mainly by lowering the fasting plasma TG concentration.

The main purpose of the present study was to examine whether exercise reduced lipaemia after a meal of moderate fat content (35\% total energy) in healthy eumenorrhoeic women. The rationale behind this question was that, as mentioned earlier, an adequacy of endogenous oestrogens has been associated with low postprandial lipaemia (Westerveld, 1998; Van Beek et al. 1999). It is thus worth asking whether exercise confers an additional benefit in this respect. This issue has not been explored as the relevant studies that have examined women as a group have not reported their menstrual status (Aldred et al. 1994; Tsetsonis \& Hardman, 1996a,b), have not controlled for the phase of the menstrual cycle (Tsetsonis et al. 1997; Herd et al. 2000; Thomas et al. 2001; Gill et al. 2002, 2003) or have employed postmenopausal women (Gill \& Hardman, 2000; Murphy et al. 2000; Koutsari et al. 2001).

Individual fatty acids play distinct biological roles, a striking example being the well-known divergent effects of saturated and unsaturated fatty acids on lipoprotein metabolism and risk of atherosclerosis. With the exception of one study from our laboratory (Kolifa et al. 2004), the effect of exercise on the postprandial fatty acid profile in either the TG or NEFA fraction has not been explored. Thus, the second aim of the present study was to investigate this effect. 


\section{Subjects and methods}

Subjects

Eight high-level female rowers, aged 18-25 years, participated in the study. Their body mass, height, BMI, and waist:hip ratio (means with their standard errors) were 66.9 (SE 2.3) kg, 1.71 (SE $0.02) \mathrm{m}, 22.9$ (SE 0.5$) \mathrm{kg} / \mathrm{m}^{2}$ and 0.74 (SE 0.02 respectively). The subjects were non-smokers, were not suffering from any apparent acute or chronic illness, were not taking any medication or dietary supplements and were eumenorrhoeic (reporting their menstrual cycle as lasting 24-30 d). Additionally, they were normolipidaemic and euglycaemic, as judged from the analysis of fasting blood samples, which they provided during a preliminary visit to the laboratory and which produced concentrations of $0.26-0.80 \mathrm{mmol} / \mathrm{l}$ for TG, $2.91-4.46 \mathrm{mmol} / \mathrm{l}$ for total cholesterol, $1.50-2.48 \mathrm{mmol} / \mathrm{l}$ for HDL-cholesterol and $4.39-5.73 \mathrm{mmol} / \mathrm{l}$ for glucose.

Subjects were informed, orally and in writing, of the design and possible risks of the study, and consented to participate. The study was designed and carried out according to the guidelines of the University of Thessaloniki Ethics Committee.

\section{Design}

Each subject took part in two trials in a random, counterbalanced design. The first trial was performed during the first week after mid-cycle, and the second trial was performed during the same time of the next cycle. Thus, both trials fell within the luteal phase, during which oestrogen concentrations are more stable and higher than during the follicular phase.

Each trial was conducted over $2 \mathrm{~d}$. In the afternoon of day 1, the subjects either exercised for $80 \mathrm{~min}$ between 16.30 and 18.00 hours or abstained from exercise. Exercise was performed in a microprocessor-controlled rowing ergometer (Concept 2, Morrisville, VT, USA) at a constant power output corresponding to $55 \%$ of each subject's maximal aerobic power. Maximal aerobic power had been determined by an all-out race test $(2000 \mathrm{~m})$ during the preliminary visit, 1-2 weeks before the first trial, in the same ergometer as was used for the bout of experimental exercise. During the same visit, maximal heart rate had been determined by a $1 \mathrm{~min}$ all-out test in the same ergometer. Heart rate was monitored by a Polar Accurex monitor (Kempele, Finland).

Subjects had dinner no later than 20.00 hours on day 1. During the morning of day 2 , subjects arrived at the laboratory after an overnight fast. A cannula was inserted into a forearm vein and, after $5 \mathrm{~min}$ rest, $5 \mathrm{ml}$ baseline blood samples were obtained with the subjects seated. Blood was transferred into precooled test tubes containing EDTA to prevent clotting and was immediately centrifuged at $1500 \mathrm{~g}$ at $4^{\circ} \mathrm{C}$ for $5 \mathrm{~min}$. Plasma was promptly removed, divided into aliquots for the determination of lipids, glucose, insulin and oestradiol, and stored at $-80^{\circ} \mathrm{C}$.

Immediately after providing the fasting blood sample, at 08.00 hours, subjects consumed a test meal within $15 \mathrm{~min}$. The meal consisted of skimmed milk, cornflakes, bread, orange juice, low-fat ham and macadamia nuts. The latter have been used to trace the entry of dietary fat into the circulation (Fielding et al. 2000) because of their unusual fatty acid profile (a high proportion of MUFA). The composition of the meal, based on the average body mass of the participants, is shown in Table 1 . The meal contained, per kg body mass, $0.66 \mathrm{~g}$ fat, $2.14 \mathrm{~g}$ carbohydrate and $0.62 \mathrm{~g}$ protein. Its energy content was $71 \mathrm{~kJ} / \mathrm{kg}$
Table 1. Composition of the test meal for a $67 \mathrm{~kg}$ subject $^{\star}$

\begin{tabular}{lrrrrc}
\hline Food & & $\begin{array}{c}\text { Fat } \\
(\mathrm{g})\end{array}$ & $\begin{array}{c}\text { Carbohydrate } \\
(\mathrm{g})\end{array}$ & $\begin{array}{c}\text { Protein } \\
(\mathrm{g})\end{array}$ & $\begin{array}{c}\text { Energy } \\
(\mathrm{kJ})\end{array}$ \\
\hline Milk & $258 \mathrm{ml}$ & 0.0 & 12.4 & 9.5 & 367 \\
Cornflakes & $48 \mathrm{~g}$ & 0.4 & 39.7 & 3.9 & 742 \\
Bread & $95 \mathrm{~g}$ & 1.8 & 47.1 & 8.0 & 991 \\
Orange juice & $335 \mathrm{ml}$ & 0.0 & 39.1 & 0.7 & 666 \\
Ham & $91 \mathrm{~g}$ & 2.0 & 2.3 & 15.4 & 371 \\
Macadamia nuts & $52 \mathrm{~g}$ & 40.1 & 2.4 & 4.1 & 1617 \\
Sum & & 44.2 & 143.1 & 41.6 & 4756 \\
\hline
\end{tabular}

*Data derived from Food Standards Agency (2002) and Nutrition Facts food labels. For details of subjects and procedures, see p. 699 of proofs.

(corresponding to that of a main daily meal), $35 \%$ being derived from fat, $50 \%$ from carbohydrate and $15 \%$ from protein.

Blood samples were drawn at $0 \cdot 5,1,2,3,4,5,6$ and $8 \mathrm{~h}$ postprandially, as described for the baseline sample. The cannula was kept patent by flushing with normal saline. Subjects remained in the laboratory during this period, sitting or lying down. They were allowed no food or drink except for water ad libitum during the first trial. Water intake was recorded and was repeated, in terms of both quantity and timing, during the second trial.

To control for the effect of prior diet on the outcome measures of the study, subjects followed individualised isoenergetic dietary plans with the same macronutrient composition as the test meal ( $35 \%$ fat, $50 \%$ carbohydrate, $15 \%$ protein) for $2 \mathrm{~d}$ before the first test meal. They were asked to record their food intake and the time of food consumption during those days and to repeat this dietary intervention before the second test meal. They were also asked to abstain from alcohol during the $2 \mathrm{~d}$ and from caffeine during the day preceding each test meal. Finally, subjects were asked to perform no exercise other than the experimental bout during the $2 \mathrm{~d}$ before each test meal. Thus, the participants abstained from exercise for at least $60 \mathrm{~h}$ before the test meal; this period has been shown to eliminate any effects of training or previous exercise on postprandial lipaemia (Hardman et al. 1998; Herd et al. 1998, 2000).

Assays

TG and NEFA were extracted from plasma after the addition of heptadecanoic acid and triheptadecanoylglycerol (both from Sigma, St Louis, MO, USA) as internal standards. They were separated by TLC, and their fatty acid content was determined by GC as previously described (Kolifa et al. 2004). Total TG were calculated as the sum of their individual fatty acids divided by 3 . The intra- and interassay $\mathrm{CV}$ for the whole analysis was $8 \%$. This method correlated very well with the usual photometric assays $(r 0.95)$. Glucose was assayed by a photometric method using a kit from BEST (Athens, Greece).

Insulin and oestradiol were determined by enzyme immunoassay using kits from DRG (Marburg, Germany). We measured oestradiol in order to verify that the participants had similar levels of the hormone during the two trials. Because eating is not known to affect the plasma oestradiol concentration, we considered it pointless to measure the hormone in all the blood samples. Therefore, we measured it at three time points $(0,4$ and $8 \mathrm{~h})$.

\section{Dietary analysis}

Dietary records were analysed in Microsoft Access (Redmont, WA, USA) by the use of a food database developed in our laboratory, based on published data (Food Standards Agency, 2002). 


\section{Calculations and statistical analysis}

As summary measures of the responses of plasma TG, NEFA, glucose and insulin to the test meal, areas under the curves (AUC) of their concentrations $v$. time were calculated using the trapezoidal rule. Additionally, an incremental AUC was calculated for TG by subtracting the area attributable to the baseline TG concentration from total the AUC. Based on the glucose and insulin AUC, an insulin sensitivity index (Belfiore et al. 2001) was calculated for each participant and each trial as:

$$
2 /(\mathrm{INS} \times \mathrm{GLU}+1)
$$

where INS and GLU are the AUC of insulin and glucose divided by the corresponding means in the control trial.

SPSS version 10.0 (SPSS Inc., Chicago, IL, USA) was used for the statistical analysis. Values are expressed as means with their standard errors. The distribution of all dependent variables was examined using the Shapiro-Wilk test and was found not to differ significantly from normal. Significant differences between exercise and rest with regard to plasma concentrations were detected by two-way (treatment $\times$ time) ANOVA with repeated measures on both factors. Significant interactions were followed up by simple main-effect analysis.

Differences between exercise and rest with regard to AUC, as well as between baseline plasma TG and the test meal with regard to percentage fatty acid composition, were examined using the Student's $t$ test. Linear correlation analysis was carried out by Pearson's product-moment correlation. The level of statistical significance was set at $\alpha=0.05$ for all analyses.

To determine the meaningfulness of the effect of exercise on the biochemical parameters measured, we calculated effect sizes as the difference between means in the exercise and control trials divided by the standard deviation of the control trial. Effect sizes were considered small, moderate and large if their absolute values were 0.2 or less, about 0.5 , and 0.8 or more, respectively (Thomas \& Nelson, 1996). Finally, observed power was computed by the statistical software and was considered adequate if it was 0.8 (Thomas \& Nelson, 1996).

\section{Results}

Heart rate during the rowing exercise bout was 165 (SE 2) beats/ min, corresponding to 81 (SE 1)\% of maximal heart rate. The power output of the rowers was 130 (SE 3) W (55\% maximal aerobic power), and the estimated net energy expenditure of exercise was 4.2 (SE 0.1) J, or 62 (SE 2) kJ/kg body mass, as calculated by the ergometer microprocessor based on a mechanical efficiency of $25 \%$. The subjects' daily energy intake during the $2 \mathrm{~d}$ preceding the trials was 7.4 (SE 0.5) MJ, with $34 \%$ derived from fat, $47 \%$ from carbohydrate and $19 \%$ from protein.

In all, thirteen fatty acids were detected in considerable amounts in the test meal, namely: laurate $(12: 0)$; myristate $(14: 0)$; palmitate $(16: 0)$; palmitoleate $(16: 1 n-7)$; stearate $(18: 0)$; oleate $(18: 1 n-9)$; cis-vaccenate $(18: 1 n-7)$; linoleate $(18: 2 n-6) ; \gamma$-linolenate $(18: 3 n-6) ; \alpha$-linolenate $(18: 3 n-3)$; gondoate (20:1n-9); arachidonate $(20: 4 n-6)$; lignocerate (24:0). Their molar distribution is presented in Table 2 . On the other hand, twelve fatty acids were detected in considerable amounts in plasma, namely: $14: 0 ; 16: 0 ; 16: 1 n-7 ; 18: 0$; $18: 1 n-9 ; 18: 1 n-7 ; 18: 2 n-6 ; 18: 3 n-6 ; 18: 3 n-3 ; 20: 1 n-9$; dihomo- $\gamma$-linolenate $(20: 3 n-6) ; 20: 4 n-6$. Their mean molar distribution in the TG of the baseline samples (pooled data
Table 2. Percentage molar fatty acid composition of the test meal and fasting plasma triacylglycerols (Values are means with their standard errors)

\begin{tabular}{lcccccc}
\hline & & \multicolumn{2}{c}{ Plasma } & & \multicolumn{2}{c}{ Meal/plasma } \\
\cline { 3 - 4 } \cline { 7 - 7 } & & & & & \\
\cline { 7 - 8 } Fatty acid & Meal & Mean & SE & & Mean & SE \\
\hline $12: 0$ & 0.31 & - & - & & - & - \\
$14: 0$ & 1.11 & 1.61 & 0.14 & & 0.78 & 0.07 \\
$16: 0$ & 10.33 & 28.01 & 0.51 & & 0.37 & 0.01 \\
$16: 1 n-7$ & 16.74 & 3.44 & 0.19 & & 5.08 & 0.26 \\
$18: 0$ & 2.94 & 3.70 & 0.17 & & 0.82 & 0.04 \\
$18: 1 n-9$ & 57.31 & 42.40 & 0.88 & & 1.36 & 0.03 \\
$18: 1 n-7$ & 3.48 & 2.57 & 0.09 & & 1.37 & 0.04 \\
$18: 2 n-6$ & 4.35 & 16.20 & 0.72 & & 0.28 & 0.01 \\
$18: 3 n-6$ & 0.02 & 0.35 & 0.05 & & 0.07 & 0.01 \\
$18: 3 n-3$ & 0.35 & 0.30 & 0.03 & & 1.35 & 0.15 \\
$20: 1 n-9$ & 2.56 & 0.30 & 0.02 & & 8.79 & 0.45 \\
$20: 3 n-6$ & - & 0.35 & 0.03 & & - & - \\
$20: 4 n-6$ & 0.12 & 0.79 & 0.07 & & 0.17 & 0.01 \\
$24: 0$ & 0.38 & - & - & & - \\
\hline
\end{tabular}

For details of subjects and procedures, see p. 699 of proofs.

from both trials) is also presented in Table 2. Fatty acids $16: 1 n-7,18: 1 n-9,18: 1 n-7$ and $20: 1 n-9$ (all MUFA) were significantly more abundant in the test meal compared with plasma TG $(P<0 \cdot 001)$, reflecting the unique fatty acid profile of macadamia nuts, which accounted for $91 \%$ of the meal's fat.

Total plasma TG concentrations at baseline and during the postprandial period with and without prior exercise are shown in Fig. 1. TG changed significantly with time $(P=0.004)$ and were lower in the exercise trial $(P=0.001)$. In the control trial, TG concentration increased gradually and plateaued at $5-6 \mathrm{~h}$ postprandially. In the exercise trial, TG concentration remained at or below baseline up to $3 \mathrm{~h}$ and then increased gradually up to $6 \mathrm{~h}$.

The AUC of TG concentration $v$. time was reduced after exercise (3.92 (SE 0.36) v. 6.05 (SE 0.61$) \quad \mathrm{mmol} / \mathrm{l} \times \mathrm{h}$, $P<0.001)$. The effect size was -1.24 and the observed power 1.00. Incremental AUC was reduced by exercise proportionally to total AUC (0.76 (SE 0.20) v. 1.19 (SE 0.33) $\mathrm{mmol} / \mathrm{l} \times \mathrm{h})$, although with lower statistical scores $(P=0 \cdot 13$, effect size -0.46 , power 0.32 ) because of the relatively high variance of values. In addition, a positive correlation was found between

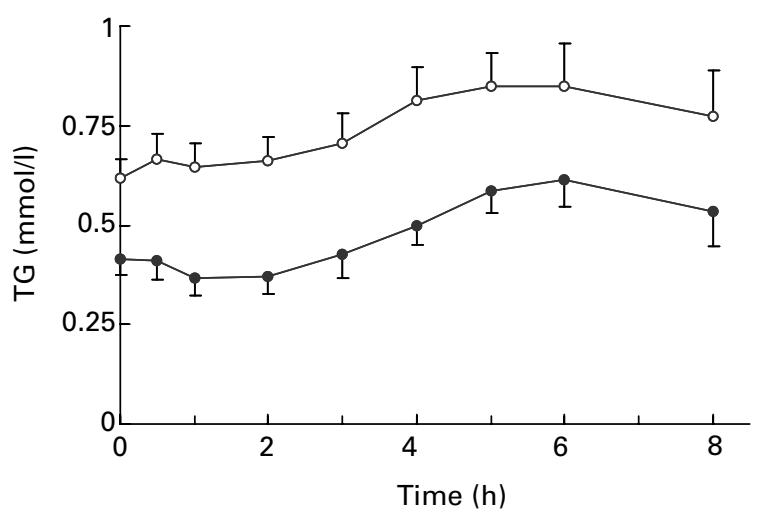

Fig. 1. Fasting $(\mathrm{O} h)$ and postprandial plasma triacylglycerol (TG) concentrations attributable to a meal of moderate fat content consumed $14 \mathrm{~h}$ after exercise $(\bullet)$ or rest $(\circ)$. Values are means and their standard errors for eight women. TG were significantly lower in the exercise trial $(P=0.001)$. For details of subjects and procedures, see p. 699 of proofs. 
the baseline TG concentration and total AUC in both trials $(r 0.80$, $P=0.02$ for exercise; $r 0.85, P=0.01$ for control) but not between baseline TG and incremental AUC.

All the individual fatty acids of plasma TG changed significantly with time and were lower in the exercise trial $(P<0 \cdot 05)$. However, their kinetics differed depending on their meal:plasma ratio (Table 1), with two patterns emerging. The four fatty acids with the highest meal:plasma ratio (i.e. $16: 1 n-7,18: 1 n-$ $9,18: 1 n-7,20: 1 n-9)$ increased gradually between 2 and $6 \mathrm{~h}$ in both trials; after this, they plateaued in the control trial and declined in the exercise trial. Fatty acid $16: 1 n-7$ typifies this pattern (Fig. 2 (A)). In contrast, the other fatty acids (i.e. 14:0, $16: 0,18: 0,18: 2 n-6,18: 3 n-6,18: 3 n-3,20: 3 n-6,20: 4 n-$ 6) peaked between 0.5 and $4 \mathrm{~h}$ and gradually declined afterwards in the control trial, whereas in the exercise trial they fell below baseline between 0.5 and $4-5 \mathrm{~h}$. Fatty acid $16: 0$ typifies this pattern (Fig. 2 (B)).

Total plasma NEFA (Fig. 3) changed significantly with time $(P<0.001)$ and were higher in the exercise trial $(P=0.007)$. There was also a treatment-by-time interaction $(P=0 \cdot 011)$. The two curves had similar kinetics up to $3 \mathrm{~h}$, but then the NEFA concentration increased abruptly in the exercise trial and was higher than control at $4-6 \mathrm{~h}(P \leq 0 \cdot 05)$. The exercise curve plateaued afterwards and met the control curve at $8 \mathrm{~h}$. The NEFA concentrations at $8 \mathrm{~h}$ were close to the baseline values. In accordance with the NEFA concentrations, the AUC of NEFA concentration $v$. time was higher after exercise (2.08 (SE 0.22) v. 1.47 (SE 0.11) $\mathrm{mmol} / \mathrm{l} \times \mathrm{h}, P=0.003$, effect size 2.01 , observed power 0.93 ).

The curves of the individual NEFA were qualitatively the same as those of total NEFA. Again, however, we noticed two patterns
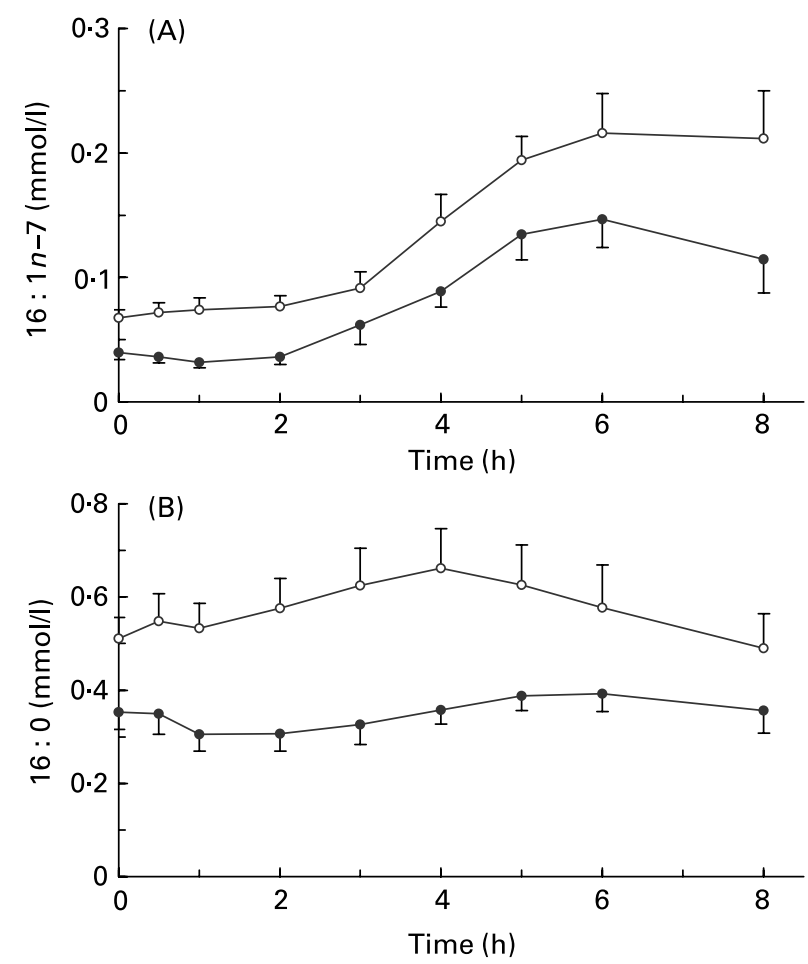

Fig. 2. Postprandial plasma kinetics of two triacylglycerol acyl groups exhibiting two distinct patterns: a gradual increase up to $6 \mathrm{~h}(\mathrm{~A})$ and a relatively stable response $(B)$. $(\circ)$ Control trial and $(\bullet)$ exercise trial. For details of subjects and procedures, see p. 701 of proofs.

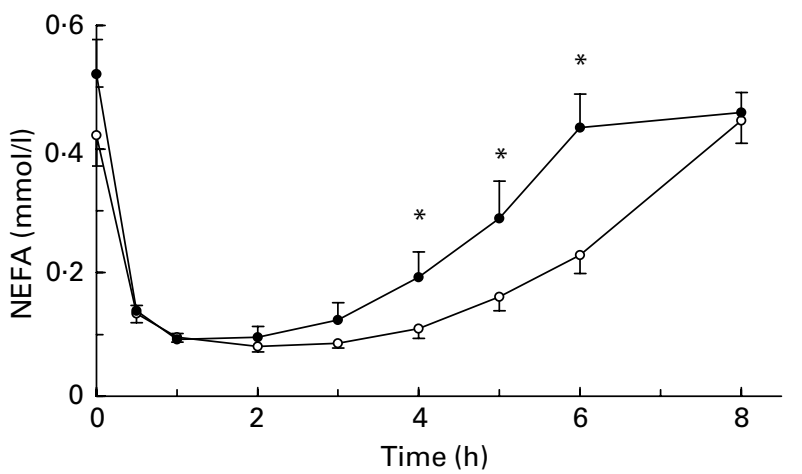

Fig. 3. Fasting and postprandial plasma concentrations of total NEFA. (०) Control trial and $(\bullet)$ exercise trial. Mean values were significantly different between the exercise and control groups; ${ }^{*} P \leq 0.05$. For details of subjects and procedures, see p. 701 of proofs.

according to the meal:plasma TG ratio of each fatty acid. Fatty acids 16:1n-7, 18:1n-7, 18:1n-9 and $20: 1 n-9$ (the fatty acids with the highest meal:plasma ratios) exceeded their baseline values at 6 and $8 \mathrm{~h}$ in both trials, whereas the remaining NEFA did not. Representative curves are shown in Fig. 4. As an index of the unsaturation state of plasma NEFA, we calculated their unsaturated:saturated ratio (U:S; Fig. 5). The exercise and control curves resembled the corresponding ones for total NEFA. There was a significant main effect of time $(P<0.001)$, main effect of treatment $(P=0 \cdot 006)$ and interaction $(P=0 \cdot 030)$. U:S was significantly higher in the exercise trial between 2 and $6 \mathrm{~h}$.

Plasma glucose concentrations (Fig. 6 (A)) changed significantly with time $(P=0.012)$ and were lower in the exercise trial
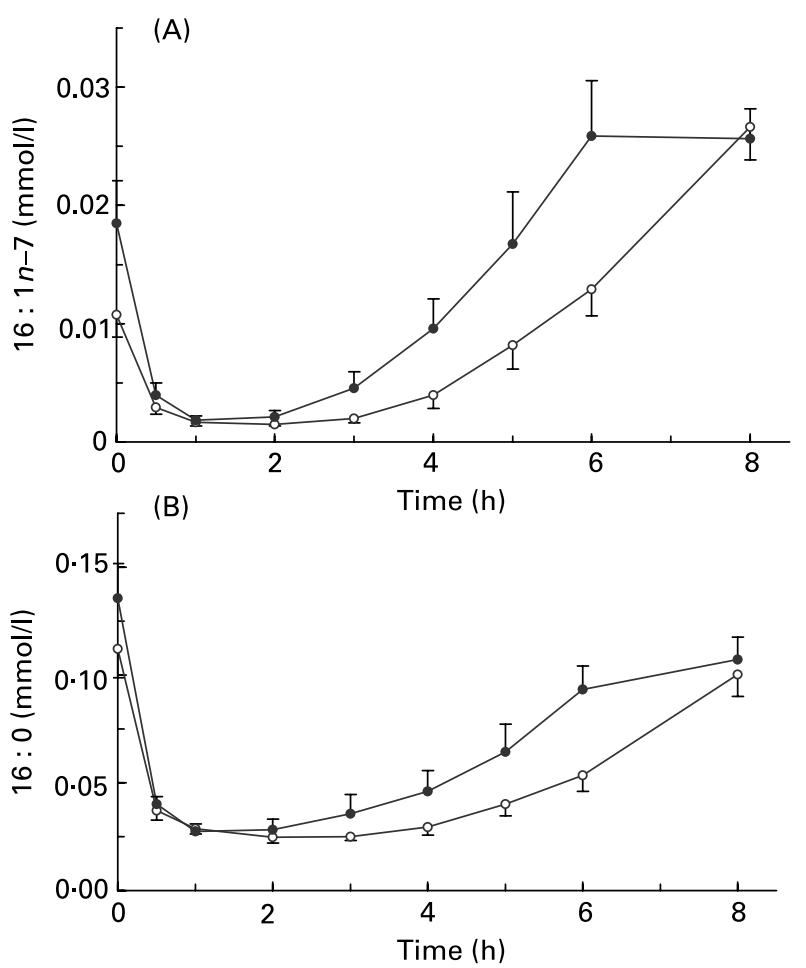

Fig. 4. Postprandial plasma kinetics of two NEFA exhibiting two distinct patterns: exceeding the baseline at 6 and $8 \mathrm{~h}(\mathrm{~A})$ and not exceeding baseline at any time point $(B) .(\circ)$ Control trial and $(\bullet)$ exercise trial. For details of subjects and procedures, see p. 699 of proofs. 


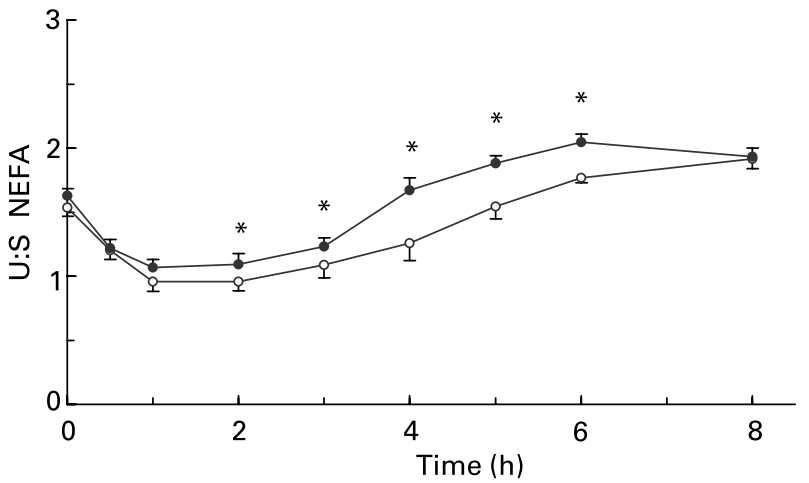

Fig. 5. Fasting and postprandial ratio of unsaturated:saturated (U:S) NEFA. $(\circ)$ Control trial and $(\bullet)$ exercise trial. Mean values were significantly different between the exercise and control groups; ${ }^{*} P \leq 0.05$. For details of subjects and procedures, see p. 699.

$(P=0.003)$. The AUC of glucose concentration $v$. time was lower after exercise $(36.7$ (SE 0.5) v. $39.4(\mathrm{SE} 0.8) \mathrm{mmol} / \mathrm{l} \times \mathrm{h}, P=0.003$, effect size $-1 \cdot 24$, observed power 0.97).

Insulin concentrations (Fig. 6 (B)) changed significantly with time $(P=0.003)$ and were lower in the exercise trial $(P=0.010)$. Both curves peaked at $0.5 \mathrm{~h}$ and then gradually decreased, reaching baseline at $5 \mathrm{~h}$. The AUC of insulin concentration $v$. time was lower after exercise (142 (SE 30) v. 173 (SE 28) $\mathrm{mU} / 1 \times \mathrm{h}$, $P=0 \cdot 003$, effect size $-0 \cdot 46$, observed power $0 \cdot 98)$.

The insulin sensitivity index, calculated from the postprandial insulin and glucose responses, was higher after exercise compared with rest (1.18 (SE 0.09) v. 1.03 (SE 0.06), $P=0.001$, effect size 1.03 , observed power 0.88). Finally, baseline plasma oestradiol concentrations did not differ significantly in the two trials (304 (SE 50) pmol/l in control v. 311 (SE 31) pmol/l in exercise con-
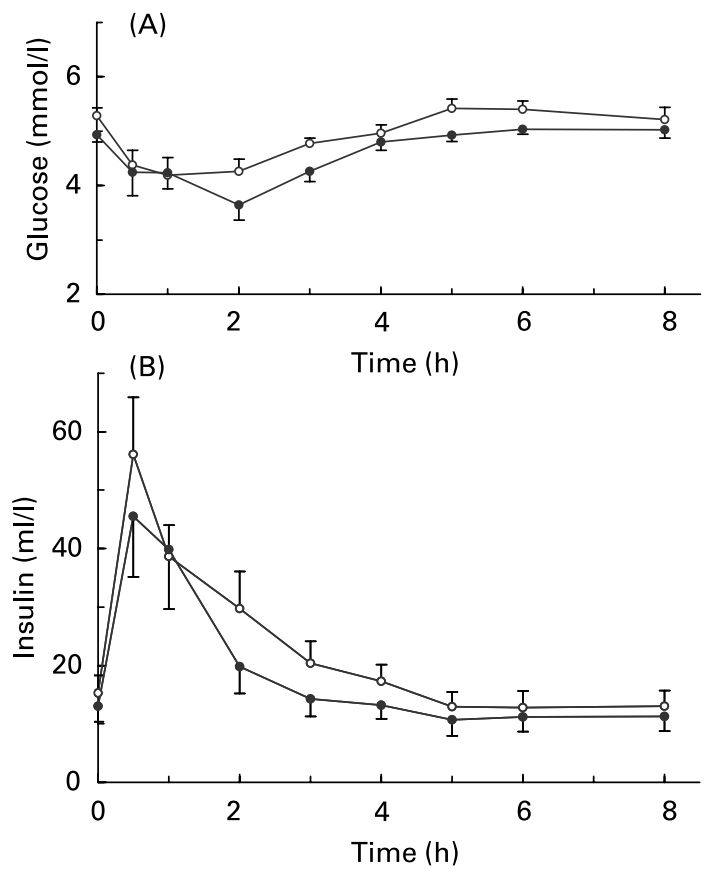

Fig. 6. Fasting and postprandial plasma glucose and insulin concentrations. $(\circ)$ Control trial and $(\bullet)$ exercise trial. For details of subjects and procedures, see p. 699. ditions) and did not change significantly in the postprandial period (data not shown).

\section{Discussion}

The main finding of the present study was that exercise reduced postprandial lipaemia after a moderate-fat meal by $35 \%$ in eumenorrhoeic women in the luteal phase. The exercise stimulus was similar, in terms of duration and intensity, to that in other relevant studies and was sufficient to elicit two well-documented acute responses: a decrease in fasting TG concentration (e.g. Thompson et al. 2000) and an increase in insulin sensitivity (e.g. Borghouts \& Keizer, 2000). The latter was the result of reductions in both the insulin and the glucose AUC with exercise (by $7 \%$ and $18 \%$, respectively).

Although a lower postprandial insulin response with exercise has also been observed in about half of the relevant studies (Tsetsonis \& Hardman, 1996a; Tsetsonis et al. 1997; Gill et al. 1998, 2001, 2002; Hardman et al. 1998; Malkova et al. 1999; Gill \& Hardman, 2000; Koutsari et al. 2001), the lower glucose response found in the present study is new. The unusual absence of a rise in the postprandial plasma glucose concentration may be due to the high basal rate of glucose uptake by the muscles of endurance-trained athletes (as were the participants in the present study) and their high insulin sensitivity (Goodyear \& Kahn, 1998). It is thus possible that the peak in the postprandial glucose - and even insulin - concentration occurred before the $30 \mathrm{~min}$ sampling point.

We have examined the effect of exercise on postprandial lipaemia in eumenorrhoeic women while controlling for a possible confounding variable (phase of the menstrual cycle) and measuring plasma oestradiol concentration, which had very similar values in the two trials. The finding that exercise was able to mitigate postprandial lipaemia in a hormonal environment, which has been reported to favour a low postprandial lipaemic response (Westerveld, 1998; Van Beek et al. 1999), suggests that it may confer an additional benefit.

Unlike most studies from other laboratories, the present study used a moderate-fat meal (35\% total energy), approximating the macronutrient composition of a typical Western diet. Again, this did not prevent exercise from having an effect on postprandial lipaemia. This finding may be more applicable to everyday life than the findings of studies with high-fat meals. Both in the present study, and in our previous study employing macadamia nuts as the main fat source (Kolifa et al. 2004), we observed a delayed postprandial peak (at 5-6h) in plasma TG concentration compared with similar studies (including another from our laboratory; Petridou et al. 2004) that used other fat sources. This may indicate a slow absorption of the fat from macadamia nuts.

The kinetics of plasma NEFA (Fig. 3) reflected the nutritional state of the subjects: NEFA concentrations dropped sharply after meal consumption, apparently because of an inhibition of hormone-sensitive lipase in adipose tissue by insulin. The subsequent rise in plasma NEFA is mainly due to the spill-over of NEFA from chylomicron TG through the action of lipoprotein lipase in the adipose tissue capillaries (Frayn, 1998). This also explains why the individual TG fatty acids that increased postprandially in both trials (Fig. 2) coincided with the individual NEFA that exceeded the fasting values at 6 and $8 \mathrm{~h}$ (Fig. 4). As with our previous relevant study (Kolifa et al. 2004), the relatively slow return 
of NEFA to fasting concentrations, compared with other studies, can be explained by the high carbohydrate content of the test meal, which resulted in higher insulin concentrations than were seen in studies with high-fat/low-carbohydrate meals.

What came as a surprise regarding the plasma NEFA kinetics was their higher concentrations and AUC (by 41\%) in the exercise trial, since most relevant studies have found no significant differences between rest and exercise (Aldred et al. 1994; Tsetsonis \& Hardman, 1996a,b; Gill et al. 1998, 2002; Herd et al. 1998, 2000; Malkova et al. 1999, 2000; Koutsari \& Hardman, 2001; Koutsari et al. 2001; Kolifa et al. 2004). The difference observed in the present study may be attributed to a higher release of NEFA from adipose tissue in the exercise compared with the control trial.

Why, however, is there a higher release of NEFA despite a lower plasma TG level? This could be due to higher lipoprotein lipase activity, higher hormone-sensitive lipase activity, decreased TG synthesis or a combination of these effects in adipose tissue. Because insulin increases lipoprotein lipase activity, decreases hormone-sensitive lipase activity and stimulates TG synthesis in adipose tissue (Frayn, 1998), and because the postprandial insulin response was lower in the exercise trial, the possibility of a higher lipoprotein lipase activity in the exercise compared with the control trial is not favoured. It is thus possible that an exercise-induced activation of hormone-sensitive lipase and/or a suppression of TG synthesis in the adipocytes created an intracellular surplus of NEFA and a more shallow concentration gradient across the plasma membrane, which directed more NEFA toward the circulation.

Another interesting finding of the present study was the higher U:S NEFA in the exercise trial. The physiological significance of a high U:S lies in the beneficial role of unsaturated fatty acids in human health. Apart from the well-established negative correlation between the intake of unsaturated fatty acids and the risk of CVD, evidence has been presented linking unsaturated fatty acids with insulin sensitivity (e.g. Gill et al. 1998). The higher $\mathrm{U}: \mathrm{S}$ NEFA in the exercise trial is probably due to the higher total NEFA concentration, since, as already mentioned, circulating NEFA in the postprandial state emanate from dietary TG, which had a U:S (5.8, as calculated from Table 2) that was higher than that of plasma NEFA (Fig. 5). No study on the effect of exercise on postprandial lipaemia except the ones from our laboratory (Kolifa et al. 2004; Petridou et al. 2004) has analysed individual fatty acids. It is worth noting that we have found a positive effect of exercise on plasma U:S NEFA in the fasted state as well (Mougios et al. 2003), although that effect lasted less than $2 \mathrm{~h}$. In contrast, the effect in this study was in the postprandial period, $16-20 \mathrm{~h}$ after exercise.

In conclusion, aerobic exercise performed $14 \mathrm{~h}$ before the consumption of a moderate-fat meal had a large reducing effect on postprandial lipaemia in eumenorrhoeic women studied in the luteal phase and controlled for serum oestradiol concentration. This shows the potential of exercise to mitigate lipaemia, and thus play a cardioprotective role, even in the face of a moderate-fat diet and eumenorrhoea, which alone are associated with a reduced risk of CVD. Exercise also attenuated the postprandial glycaemic and insulinaemic responses, whereas it increased the NEFA response, suggesting a lower entrapment of dietary fatty acids in adipose tissue. Finally, exercise increased the proportion of U:S NEFA during the postprandial period, which may confer an additional health benefit.
From the positive findings of our two studies with moderate-fat meals (the present one and that of Kolifa et al. 2004), it appears that high-fat meals may not be necessary for exercise to reduce postprandial lipaemia. We therefore suggest that future studies in this field should focus on the characterisation of the effects of exercise on lipaemia after meals of a regular fat content. Apart from increasing the applicability of the findings to everyday life, this will facilitate the harmonisation of physical activity recommendations with dietary recommendations for healthier living.

\section{Acknowledgements}

We thank Sakis Athanasiadis, who was the participants' coach, for his valuable help with the exercise protocols. This work was supported by a donation from the Bodossaki Foundation.

\section{References}

Aldred HE, Perry IC \& Hardman AE (1994) The effect of a single bout of brisk walking on postprandial lipemia in normolipidemic young adults. Metabolism 43, 836-841.

Belfiore F, Iannello S, Camuto M, Fagone S \& Cavaleri A (2001) Insulin sensitivity of blood glucose versus insulin sensitivity of blood free fatty acids in normal, obese, and obese-diabetic subjects. Metabolism 50, $573-582$.

Borghouts LB \& Keizer HA (2000) Exercise and insulin sensitivity: a review. Int J Sports Med 21, 1-12.

Fielding BA, Reid G, Grady M, Humphreys SM, Evans K \& Frayn KN (2000) Ethanol with a mixed meal increases postprandial triacylglycerol but decreases postprandial non-esterified fatty acid concentrations. Br J Nutr 83, 597-604.

Food Standards Agency (2002) McCance and Widdowson's The Composition of Foods. Cambridge: Royal Society of Chemistry.

Frayn KN (1998) Non-esterified fatty acid metabolism and postprandial lipemia. Atherosclerosis 141, S41-S46.

Gill JMR \& Hardman AE (2000) Postprandial lipemia: effects of exercise and restriction of energy intake compared. Am J Clin Nutr 71, $465-471$.

Gill JMR \& Hardman AE (2003) Exercise and postprandial lipid metabolism: an update on potential mechanisms and interactions with high-carbohydrate diets. J Nutr Biochem 14, 122-132 [review].

Gill JMR, Herd SL \& Hardman AE (2002) Moderate exercise and postprandial metabolism: issues of dose-response. J Sports Sci 20, 961-967.

Gill JMR, Herd SL, Vora V \& Hardman AE (2003) Effects of a brisk walk on lipoprotein lipase activity and plasma triglyceride concentrations in the fasted and postprandial states. Eur J Appl Physiol 89, 184-190.

Gill JMR, Mees GP, Frayn KN \& Hardman AE (2001) Moderate exercise, postprandial lipaemia and triacylglycerol clearance. Eur J Clin Invest 31, 201-207.

Gill JMR, Murphy MH \& Hardman AE (1998) Postprandial lipemia: effects of intermittent versus continuous exercise. Med Sci Sports Exerc 30, 1515-1520.

Goodyear LJ \& Kahn BB (1998) Exercise, glucose transport, and insulin sensitivity. Anпu Rev Med 49, 235-261.

Hardman AE, Lawrence JEM \& Herd SL (1998) Postprandial lipemia in endurance-trained people during a short interruption to training. $J \mathrm{Appl}$ Physiol 84, 1895-1901.

Herd SL, Hardman AE, Boobis LH \& Cairns CJ (1998) The effect of 13 weeks of running training followed by $9 \mathrm{~d}$ of detraining on postprandial lipaemia. Br J Nutr 80, 57-66.

Herd SL, Lawrence JEM, Malkova D, Murphy MH, Mastana S \& Hardman AE (2000) Postprandial lipemia in young men and women of contrasting training status. J Appl Physiol 89, 2049-2056. 
Karpe F (1999) Postprandial lipoprotein metabolism and atherosclerosis. J Intern Med 246, 341-355.

Kolifa M, Petridou A \& Mougios V (2004) Effect of prior exercise on lipemia after a meal of moderate fat content. Eur J Clin Nutr 58, 1327-1335.

Koutsari C \& Hardman AE (2001) Exercise prevents the augmentation of postprandial lipaemia attributable to a low-fat high-carbohydrate diet. Br J Nutr 86, 197-205.

Koutsari C, Karpe F, Humphreys SM, Frayn KN \& Hardman AE (2001) Exercise prevents the accumulation of triglyceride-rich lipoproteins and their remnants seen when changing to a high-carbohydrate diet. Arterioscler Thromb Vasc Biol 21, 1520-1525.

Malkova D, Evans RD, Frayn KN, Humphreys SM, Jones PRM \& Hardman AE (2000) Prior exercise and postprandial substrate extraction across the human leg. Am J Physiol Endocrinol Metab 279, E1020-E1028.

Malkova D, Hardman AE, Bowness RJ \& Macdonald IA (1999) The reduction in postprandial lipemia after exercise is independent of the relative contributions of fat and carbohydrate to energy metabolism during exercise. Metabolism 48, 245-251.

Mougios V, Ring S, Petridou A \& Nikolaidis MG (2003) Duration of coffee- and exercise- induced changes in the fatty acid profile of human serum. J Appl Physiol 94, 476-484.

Murphy MH, Nevill AM \& Hardman AE (2000) Different patterns of brisk walking are equally effective in decreasing postprandial lipaemia. Int $J$ Obes 24, 1303-1309.

Petitt DS, Arngrímsson SA \& Cureton KJ (2003) Effect of resistance exercise on postprandial lipemia. J Appl Physiol 94, 694-700.

Petridou A, Gerkos N, Kolifa M, Nikolaidis M, Simos D \& Mougios V (2004) Effect of exercise performed immediately before a meal of moderate fat content on postprandial lipaemia. Brit $J$ Nutr 91, $683-687$.
Pirro M, Lupattelli G, Siepi D, Polumbo B, Roscini AR, Marchesi S, Schillaci G \& Mannarino E (2001) Postprandial lipemia and associated metabolic disturbances in healthy and hyperlipemic postmenopausal women. Metabolism 50, 330-334.

Romon M, Le Fur C, Lebel P, Edme JL, Fruchart JC \& Dallongeville J (1997) Circadian variation of postprandial lipemia. Am J Clin Nutr 65, 934-940.

Thomas JR \& Nelson JK (1996) Research Methods in Physical Activity. Champaign, IL: Human Kinetics.

Thomas TR, Horner KE, Langdon MM, Zhang JQ, Krul ES, Sun GY \& Cox RH (2001) Effect of exercise and medium-chain fatty acids on postprandial lipemia. J Appl Physiol 90, 1239-1246.

Thompson AL, Lim-Fraser M-C, Kraegen EW \& Cooney GJ (2000) Effects of individual fatty acids on glucose uptake and glycogen synthesis in soleus muscle in vitro. Am J Physiol Endocrinol Metab 279, E577-E584.

Tsetsonis NV \& Hardman AE (1996a) Effects of low and moderate intensity treadmill walking on postprandial lipaemia in healthy young adults. Eur J Appl Physiol 73, 419-426.

Tsetsonis NV \& Hardman AE (1996b) Reduction in postprandial lipemia after walking: influence of exercise intensity. Med Sci Sports Exerc 28, $1235-1242$.

Tsetsonis NV, Hardman AE \& Mastana SS (1997) Acute effects of exercise on postprandial lipemia: a comparative study in trained and untrained middle-aged women. Am J Clin Nutr 65, 525-533.

Van Beek AP, de Ruijter-Heijstek FC, Erkelens DW \& de Bruin TW (1999) Menopause is associated with reduced protection from postprandial lipemia. Arterioscler Thromb Vasc Biol 19, 2737-2741.

Westerveld HE (1998) Estrogens and postprandial lipid metabolism. Atherosclerosis 141, S105-S107. 